\title{
La educación superior. Caballo \\ de troya: ¿gubernamentalidad \\ o autonomía?*
}

\section{Revista Colombiana \\ Artículos temáticos $\quad \begin{aligned} & \text { de Educación, N. . } 65 . \\ & \text { Segundo semestre de 2013, }\end{aligned}$ \\ Bogotá, Colombia.}

\section{//Higher education: a trojan horse. \\ Governmentality or autonomy?}

//O ensino superior. Cavalo de troia:
governamentalidade ou autonomia?

Recibido: 13/06/2013

Jorge Antonio Herrera Llamas**

Texto realizado como producto de la investigación: Economía de la Educación, Política Pública y Financiamiento de la Educación Superior en Colombia. Tesis Doctoral.

Doctor en Ciencias de la Educación, Magíster en Desarrollo Económico. Docente de la Universidad de Cartagena, Docente Universidad San Buenaventura Cartagena. Correo electrónico: jaherrerallamas@gmail.com

\section{Resumen}

Desde la perspectiva de la teoría del Capital Humano el artículo presenta un análisis crítico del manejo que se le ha dado a la educación superior, mostrando cómo el discurso académico del sistema constituye el señuelo que convierte a la educación en el vehículo del modelo económico, mercantilizando a la educación superior en detrimento de la autonomía universitaria como magna expresión de la otrora Alma Máter.

De esta manera, la lucha por los mercados pone a prueba las estrategias administrativas que sin herir susceptibilidades, puedan lograr sus cometidos de dominio haciendo sentir entre los gobernados que realmente participan de las decisiones de la llamada economía del conocimiento. En este contexto, los atributos de docencia, investigación y proyección social, como funciones sustantivas de la universidad, sucumben ante las fuerzas del mercado y sus externalidades negativas.

\section{Abstract}

From the perspective of human capital theory, this article presents a critical analysis of the ways in which higher education has traditionally been managed. It shows how academic discourse around the system acts as a decoy which makes education a vehicle for embedding the economic model, modifying higher education to the detriment of university autonomy as the great expression of the former Alma Mater.

Consequently, the struggle for markets puts to test management strategies which, without causing offence, are able to achieve their task of control and domination, which is felt among the governed who are really involved in the decisions of the so-called knowledge-based economy. In this context, the attributes of teaching, research and social outreach as substantive functions of the university succumb to market forces and their negative externalities.

\section{Resumo}

Segundo a perspectiva da teoria do Capital Humano o artigo apresenta uma análise crítica da forma como se tem conduzido a educação superior, mostrando como o discurso acadêmico do sistema constitui a isca que converte a

\section{Palabras Clave}

Autonomía, capital humano, gubernamentalidad, mercado, modelo económico.

\section{Keywords}

Autonomy, human capital, governmentality, market, economic model.

\section{Palavras chave}

Autonomia, capital humano, governamentalidade, mercado, modelo econômico. 
educação no veículo do modelo econômico, mercantilizando a educação superior em detrimento da autonomia universitária como magna expressão da outrora Alma Mater.

Dessa maneira, a luta pelos mercados põe a prova as estratégias administrativas que sem ferir susceptibilidades, podem conseguir os seus propósitos de domínio fazendo acreditar aos governados que verdadeiramente participam das decisões da chamada economia do conhecimento. Nesse contexto, os atributos de ensino, pesquisa e extensão enquanto funções próprias da universidade, sucumbem diante as forças do mercado e as suas externalidades negativas.

\section{Introducción}

La utopía del ayer se convirtió en la sociedad contemporánea, en este mundo sin fronteras donde los sueños por muy grande que parezcan se convierten en el día a día del hombre moderno.

La lucha por el mercado, una batalla sin cuartel, con la convicción de la existencia de ganadores y perdedores -juego de suma cero-, crea un escenario propicio solo para emprendedores: los líderes. Es que con este espejismo administrativo se ha moldeado la conciencia social, con la promesa de encontrar en la globalización, en la lógica del mercado y en la inmediatez del relativismo económico, la respuesta mágica al ordenamiento social. Cambios psicosociales experimentados como resultado de la exposición a culturas disímiles y a patrones de consumos importados a ultranza, definen las características de una época de cambios calificada por algunos como posmodernidad (Garay, 1999).

De Europa llegan lecciones de cooperación, sentimientos transnacionales, donde el individualismo y la noción de soberanía ceden paso a la unión generadora de sinergia. Japón recuerda que existe un Dios en cada hombre de bien, por eso se levanta justo a tiempo, para enfrentar el poderío económico de Estados Unidos en el presente milenio.

La falacia sobre los conceptos de autonomía, libertad y autodeterminación garantiza al paradigma económico el dominio, no solo del sujeto como individuo componente del entramado social, sino que los mensajes prometedores de seguridad y de mejoramiento de vida se vuelven subliminales a toda la población. Herir sin dolor, dominar mediante la autoentrega (let it be) es la estrategia del modelo que soslaya el conflicto abierto, manteniendo estándares de tolerancia social que actúan como colchones de protección ante cualquier amenaza al sistema (Habermas, 1973).

La ciencia, las aplicaciones tecnológicas y lo imprescindible de la innovación, como imperativo lógico de la posmodernidad, constituyen banderas de retroalimentación continua de la llamada 
sociedad del conocimiento cuyo centro de gravitación es el mercado. El biopoder entonces, se matiza en un entramado sociojurídico donde la razón económica genera razón de Estado. Las sociedades transitan en el tiempo sin ningún horizonte seguro definido, puesto que en el dominio de lo mediato y la improvisación de decisiones para diferenciar estilos de gobernar, solo existen políticas de gobierno cada vez más penetradas por intereses corporativistas, mas no de Estado. En ese sentido, Foucault (2008) manifiesta:

El arte de gobernar debe fijarse entonces sus reglas y racionalizar sus maneras de obrar, proponiéndose en cierto modo como objetivo transformar en ser el deber ser del Estado. El deber hacer del gobierno tiene que identificarse con el deber ser del Estado (p. 19).

Sin embargo, la penetración transnacional de los poderes e intereses corporativistas no solo minan sino que redefinen el deber ser del Estado ante lo imperativo de la ecuación costos/beneficios. Este escenario, con peculiares formas de gobernanza, le impone nuevas funciones a la educación (Veiga-Neto, 2010).

Analizar la relación entre la educación superior y los modelos económicos que la utilizan para introducir sus postulados, dándole un carácter instrumental en un contexto histórico, tiene una utilidad práctica: mostrar cómo se afecta el concepto de autonomía frente a unos derroteros que obedecen a la lógica de los mercados. Al someter el análisis a la racionalidad de la ciencia económica y encontrar en los fundamentos de la política educativa una clara injerencia de los modelos y paradigmas económicos que sustentan su contenido teórico, inexorablemente surge el interrogante: ¿existe realmente autonomía universitaria o en la práctica se le da un carácter instrumental mediante la gubernamentalidad del paradigma como estrategia de crear la sensación de autogobierno bajo el influjo de la economía de mercado?

La relevancia social y científica de este artículo radica en aportar una visión económica al análisis de la educación superior. En la práctica, la teoría del capital humano toma a la educación como un sistema con input y output de insumos y productos, en este sentido, la universidad es afectada por variables endógenas y exógenas que interactúan retroalimentando el modelo mercantil.

\section{La piedra angular del sistema}

En las postrimerías del siglo XX, el animal político de Aristóteles se convirtió en el animal económico del sistema de economía de mercado. Foucault introduce el concepto de economía política como limitador de la razón del Estado, para referirse al fenómeno mediante el cual la acción de los gobernantes encuentra una 
frontera en los asuntos económicos y que en la práctica el poder económico genera poder político, esto tiene antecedentes históricos que conviven con el tiempo, lo que definió como reduccionismo histórico, en el que los conceptos universalmente aceptados dominan el presente (Foucault, 2008).

Foucault en su clase del 10 de enero de 1979, en El Colegio de Francia, plantea cómo en los siglos XVII y XVIII el derecho frenaba o limitaba la acción indefinida del Estado; en otras palabras, modulaba la acción gubernamental, pero las evidencias muestran que en la actualidad, la misma práctica jurídica se amolda para garantizar un nuevo instrumento con mayor poder regulador y de limitación del accionar del Estado: el mercado.

Desde los fundamentos de la teoría del capital humano, la educación es vista como una inversión con unos costos directos y de oportunidad definidos con claridad, en función de una rentabilidad esperada. La racionalidad economicista prima sobre cualquier criterio académico, o sea, el dominio de lo que Foucault (2008) entiende como economía política al manifestar:

Pero la cuestión económica siempre va a plantearse en el interior del campo de la práctica gubernamental y en función de sus efectos, no de lo que podría fundarla en términos de derecho: ¿cuáles son los efectos reales de la gubernamentalidad al cabo mismo de su ejercicio? Y no: ¿cuáles son los derechos originarios que pueden fundar esa gubernamentalidad? (p. 32).

El desvío de la relación universidad/sociedad justifica el carácter utilitarista que se le ha dado a la educación, en particular a la superior. Se desdeñan su origen, sus objetivos y misión como generadora de conocimiento a cambio de convertir a las universidades en fábrica de mano de obra que abastece un mercado con el sofisma de la pertinencia. En el discurso academicista la pertinencia es entendida como la diferencia entre lo que la sociedad espera de las universidades y lo que las universidades hacen en el cumplimiento de sus objetivos misionales (Unesco, 1980). Las dificultades comienzan desde la misma concepción normativa que se tiene de lo que debería ser el saber superior; de esta manera, en el caso colombiano, la educación superior no está claramente definida. La Ley 30 de 1992 plantea:

La educación superior es un proceso permanente que posibilita el desarrollo de las potencialidades del ser humano 
de una manera integral, se realiza con posterioridad a la educación media o secundaria y tiene por objeto el pleno desarrollo de los alumnos y su formación académica o profesional (art. 1).

Como es evidente, su conceptualización es ambigua y general. En este marco de ideas se desprenden diferentes ámbitos que en la práctica no delimitan sus radios de acción. De esta manera, compiten por los mismos estudiantes y con el mismo currículo lo técnico, tecnológico y lo profesional, los cuales ofrecen centros de estudios que se presentan como: instituciones técnicas profesionales, instituciones universitarias o escuelas tecnológicas y universidades.

En la "Conferencia mundial sobre educación superior en el siglo XXI: visión y acción", realizada en París en 1998 con la tutela de la Organización de las Naciones Unidas para la Educación la Ciencia y la Cultura (Unesco) se resaltó el nuevo papel que cumplía la educación en el desarrollo cultural y socioeconómico. En esos momentos se tomó la gran demanda de este tipo de educación como algo positivo, pero no se tuvo en cuenta la poca capacidad de los Estados para enfrentar estos nuevos requerimientos

Los adelantos tecnológicos se desarrollaban al ritmo de las nuevas ramas industriales y al dinamismo de los mercados que reclamaban un capital humano altamente competitivo. Así se incubó la noción de competencias en la educación superior no como estrategia para medir objetivos académicos concebidos con anterioridad en los planes docentes, sino como una forma de darle el carácter utilitarista a la educación poniéndola como incubadora del modelo económico.

La estrategia consistió en despertar el orgullo patrio de los pueblos recordando la necesidad de lograr un desarrollo autónomo, endógeno y sustentable que le permitiera por fin cerrar la brecha que se había abierto con mayor fuerza en la segunda mitad del siglo XX al unísono de la expansión de la educación superior. Ante este desafío se dio la largada de la carrera expansionista en la educación superior revestida del ideal de autonomía académica, administrativa y financiera. Entonces, ¿dónde estaba la falla?

Frente a la diferencia en los indicadores sociales y económicos entre los países industrializados y los Ilamados países en vías de desarrollo, de nuevo se enarbolaron las banderas de la educación como instrumento necesario para disminuir tales distancias ${ }^{1}$. Lo contradictorio fue que las políticas de entonces vieron la solución en lo cuantitativo del fenómeno en detrimento de la calidad e introdujeron a los países en la

1 Las cifras fueron creciendo pero la brecha entre lo rico y lo pobre no cedía y el tecnicismo ha originado que ahora los países pobres no son llamados en vía de desarrollo sino economías emergentes. 
carrera del incremento de cobertura de educación superior sin ninguna planificación, como ocurrió en Colombia (Gráfico 1).

Gráfico 1. Matrícula en educación superior (Colombia)

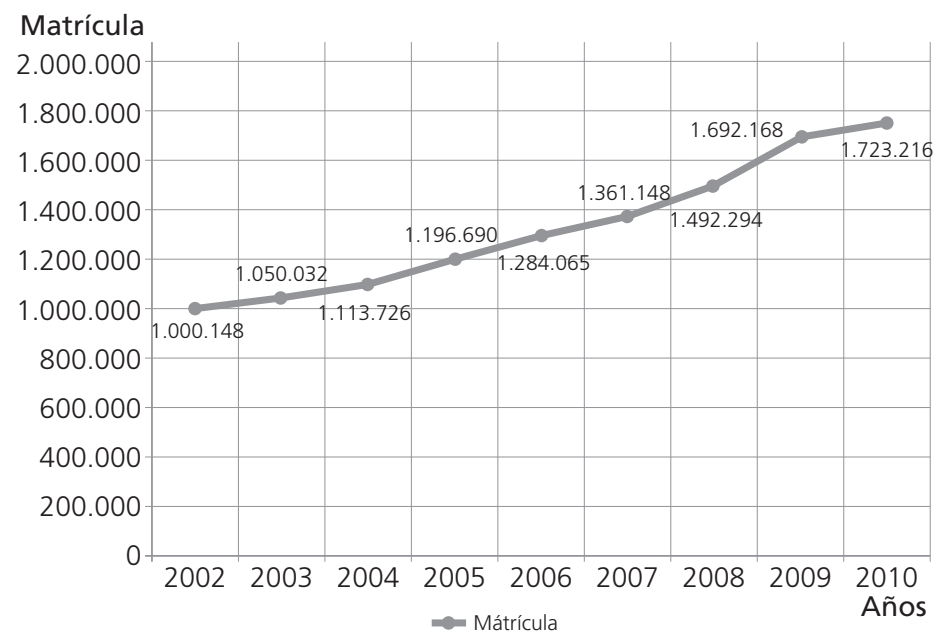

Fuente: Elaboración propia de datos del Ministerio de Educación Nacional.

En tales circunstancias se requería de una redefinición de los estudios superiores y de las instituciones con capacidades legales y académicas para ofrecerlas, con la promesa de que la única forma de salir de la pobreza era generando nuevo conocimiento, reconociendo que la educación es uno de los pilares fundamentales de los derechos humanos, la democracia, el desarrollo sostenible y la paz, y con la convicción de que la solución de los problemas del futuro dependería del papel que se le diera a la educación en general y a la educación superior en particular.

Los organismos multilaterales como la Organización de las Naciones Unidas (ONU) el Banco Mundial (BM) y el Fondo Monetario Internacional (FMI) ante la evidencia de la coexistencia de niveles económicos y sociales asimétricos que mostraban la dualidad económica en las sociedades más atrasadas, reivindicaron el concepto de la educación como un instrumento para vencer la pobreza que azotaba gran parte del mundo. En estas condiciones el modelo propugnó por cambios en los sistemas educativos, garantizando de esta manera un trampolín para adecuar de la mejor manera posible a todas las esferas del aparato estatal poniéndolo acorde con las exigencias 
liberadoras de los nuevos tiempos (Agüero, 2010). En efecto, el BM en su proceso de utilizar la educación superior para aclimatar el modelo mercantil y las nuevas formas de poder, publicó los siguientes documentos:

1. La educación superior: lecciones derivadas de la experiencia (1994).

2. La educación superior en los países en desarrollo: peligros y promesas (2000).

3. Construir sociedades de conocimiento: nuevos retos para la educación terciaria (2003).

La nueva economía ingrávida, inmersa en un proceso de globalización, requería de instrumentos que viabilizaran sutilmente los elementos de dominio de la economía de mercado. Es en este contexto donde la educación representa el vehículo más adecuado para minar la voluntad de los pueblos fascinados con los adelantos tecnológicos y con la creencia de pertenecer a la nueva sociedad del conocimiento (Rifkin, 2000). Lo anterior justificó que el paradigma se revistiera con un acentuado interés por la educación superior como motor que catapultaría a los países a la era global. Por tanto, se hacía necesario instaurar parámetros o estándares internacionales que facilitaran el proceso de integración transnacional; así se avivaron de nuevo las banderas de las homologaciones, convalidaciones, movilidad docente-estudiantil y el ideal de una educación universitaria autónoma, como magna expresión de la libertad de cátedra, dentro de la enseñanza libre (Paniagua, 2003).

La comunidad académica creyó que con la expedición de la Ley 30 de 1992 se habían logrado reivindicaciones al estilo de la reforma de 1918 en la Universidad de Córdoba, Argentina. Los vientos de autonomía, libertad de cátedra y cogobierno, atributos con que se vendió el experimento, escondieron los conceptos antinómicos del modelo, lo que Edgar Morín (2000) llama las cegueras paradigmáticas, al expresar:

El juego de la verdad y del error no solo se juega en la verificación empírica y la coherencia lógicas de las teorías; también se juega a fondo en la zona invisible de los paradigmas. Esto lo debe tener bien en cuenta la educación (p. 28).

La autonomía universitaria, consagrada en la Constitución y la ley, dotaba a la universidad pública de independencia para administrarse, darse sus propios estatutos, otorgar títulos, escoger libremente a sus autoridades y arbitrar sus recursos para el cumplimiento de su misión socia. Lo incomprensible fue que sometió el financiamiento de la universidad al comportamiento de la inflación y 
al crecimiento económico del país. Es aquí donde está la trampa económica del sistema, puesto que sus mismas directrices instaban al Estado a que dentro de un régimen de austeridad se controlaran los niveles de inflación, condenando a la asfixia financiera a la universidad y, por consiguiente, generando una autonomía espuria.

En efecto, con la implementación de las políticas económicas emanadas del Consenso de Washington, referentes a disciplina presupuestal, austeridad fiscal y prioridad del gasto público, los niveles de inflación bajaron notoriamente al pasar de 25,13\% en 1992 a 8,75\% en 2000, y 2,24\%, en 2012. La paradoja de esta política radica en que lo que al parecer era positivo para la economía, condenaba a constantes déficits a las universidades que veían depreciarse los aportes del Gobierno nacional y de los demás entes territoriales (Villarroel, 2006).

La suerte de las casas de estudios superiores empezó a depender de la capacidad que tuvieran para generar sus propios recursos; en consecuencia, la función de extensión como lazo entre la comunidad y el quehacer en las aulas universitarias fue reemplazada, sin ningún prejuicio académico, por la venta de servicios; la generación de conocimiento como invocación de la universidad humboldtiana cedió paso a las modernas fábricas de mano de obra y a un credencialismo exasperado (Clark, 1997). Los interrogantes aún quedan por responder; respuestas que no se puede encontrar en lo que Theodore Shultz en 1961 Ilamó la hipocresía académica.

En las postrimerías del siglo XX, la demanda por la educación superior se había disparado, develando la incapacidad que tenía el Estado para responder con una oferta apropiada a tal fenómeno sin precedente. Los planes de gobierno se quedaron en el corto plazo o periodos gubernamentales, incapaces de anticiparse a los resultados que en el largo plazo arrojarían el aumento en la educación básica secundaria, el afán desmedido de cumplir con los requerimientos internacionales de la universalización educativa y el ritmo demográfico nacional. Pero no solo se trató de un problema de desequilibrio entre la demanda y la oferta de educación superior, la desarticulación entre los diferentes niveles del sistema desembocó en el deterioro creciente de la calidad (Cubillos, 1998).

Esta nueva situación viabilizó el siguiente paso para que los principios de gubernamentalidad se aplicaran y, en medio de una simbiosis de autonomía y libertad, se dio paso al sector privado en la educación que se posicionó de los nichos de mercado más rentables con todo un mosaico de instituciones a granel. (Gráfico 2). 
Gráfico 2. Crecimiento de instituciones de educación superior

Instituciones

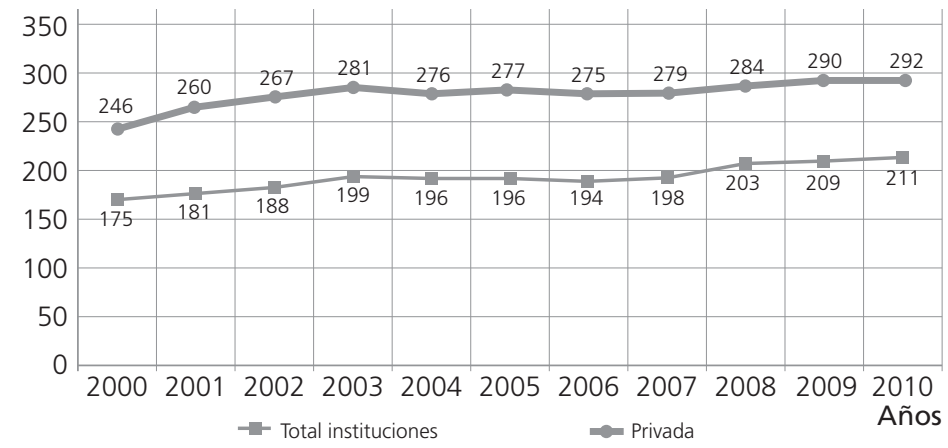

Fuente: Elaboración propia con datos de Ministerio de Educación Nacional.

Ante este comportamiento del naciente mercado universitario, el BM (2000) expresó:

Esta diversificación -que es una reacción a la mayor demanda- ha atraído al sistema a nuevos proveedores (especialmente del sector privado), y ha instado a la creación de nuevos tipos de instituciones. Ello hace prever que habrá cada vez mayor competencia y en último término, mejor calidad (p. 13).

Tomar a la educación superior como motor del desarrollo económico y social, si bien es cierto que disparó las estadísticas sobre el total de matrícula terciaria en el mundo al pasar de 13 millones de estudiantes en 1960 a 82 millones en 1995, aún se mantenía una deuda con la población demandante. Ejemplo de este déficit se puede citar a Colombia donde la cobertura en este nivel solo alcanzaba $21,75 \%$ en 1998. En consecuencia, el reto seguía vigente y los gobiernos recibirían al siglo XXI con una serie de medidas que involucraban los conceptos de cobertura, calidad y pertinencia, pero el déficit continúa como saldo en rojo y 25 años más tarde, según el Ministerio de Educación Nacional, Colombia tiene una cobertura de $42 \%$ comparada con la de República de Corea (103,9\%); Finlandia (91,6\%); Estados Unidos (89,1\%); Eslovenia $(86,9 \%) ;$ Nueva Zelandia $(82,7 \%)$; países miembros de la Organización para la Cooperación y el Desarrollo Económico (OCDE) a la que Colombia aspira a pertenecer.

El camino a seguir ante esa situación fue el más fácil, la estrategia consistió en ampararse en el artículo 26 de la Declaración Universal de los Derechos Humanos que involucra el concepto de meritocracia para garantizar el acceso a los estudios terciarios a toda la población 
en igualdad de condiciones, sin atender a elementos raciales, religiosos o económicos, por lo que se deduce que la educación sirve también para medir oportunidades y reconocer méritos. Bajo esta perspectiva de apariencia redistributiva se estaba legitimando la desigualdad merecida (Herrera, 2013).

Al involucrar el concepto de mérito para el acceso de la educación superior, invocando el mandato de la Declaración Universal de los Derechos Humanos que dictamina: "el acceso a los estudios superiores será igual para todos en función de los méritos respectivos" (art. 26), se parte del principio de que todos los aspirantes están en igualdad de condiciones para competir por los pocos cupos que ofrece el sistema de educación superior estatal; no obstante las acentuadas diferencias económicas y sociales que no garantizan igualdad en la libertad para elegir futuro como principio de desarrollo económico (Sen, 2000).

Es preciso hacer énfasis en que ante la impotencia de los Estados para corresponder con la demanda creciente de la educación terciaria, que el modelo definía como generadora de progreso y bienestar social, se acudió al principio de la meritocracia y de la apertura al sector particular, lo que justificó de alguna manera su falta de planeación y de implementación de una verdadera política de educación superior a largo plazo. Estos vacíos administrativos fueron ocupados por la oferta de cupos de instituciones particulares (Gráfico 3).

Gráfico 3. Matrícula por sector (educación superior)

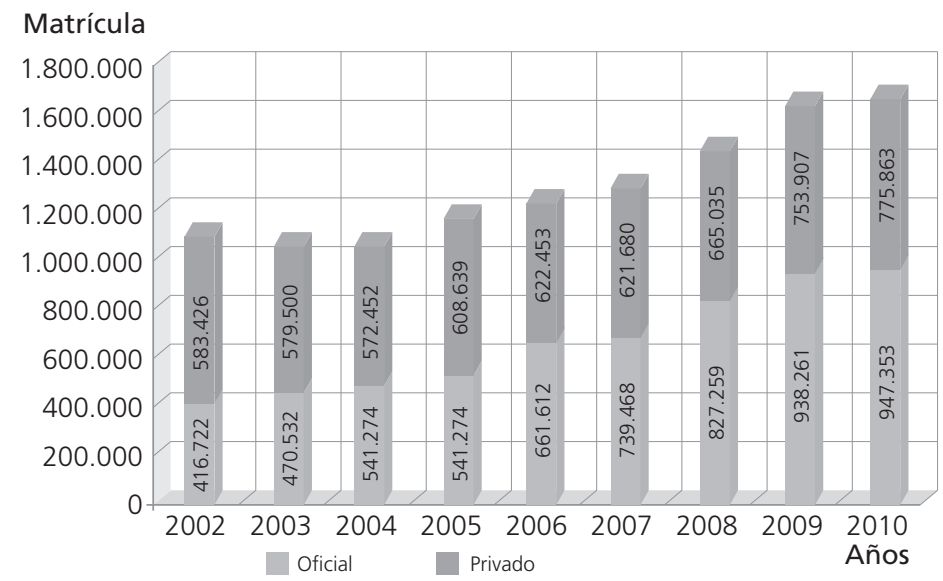


La concepción economicista de la educación, que desde la década del sesenta se trae como fundamento de la teoría del capital humano, concibe la educación como una inversión, lo que implica unos costos y una rentabilidad esperada en el futuro y la cual tiene dos connotaciones: beneficios individuales y beneficios sociales. El problema nace al considerar que la educación básica por su propia esencia es generadora de mayores beneficios sociales mientras que en la educación superior predomina la utilidad individual. Como se observa de nuevo una visión con apariencia de verdad irrebatible es utilizada para legitimar la responsabilidad financiera del sistema educativo que frente a esta lógica el Estado debía financiar la educación básica, a la vez que en los estudios superiores debía participar económicamente el individuo o beneficiario (Becker, 1983).

La teoría del capital humano aplicada a la educación debe ser revaluada en los actuales momentos, puesto que en la primera mitad del siglo XX los estudios universitarios eran privilegios de una clase, por lo general, de élite, ya que no se justificaba la inversión de recursos públicos en este tipo de educación, visión no acorde con las actuales dinámicas o concepción de la educación superior aunque dichos logros no sean tenidos en cuenta para el cálculo del índice de desarrollo humano $(\mathrm{IDH})^{2}$.

2 El índice de desarrollo humano (IDH) es el indicador utilizado por la ONU para medir el grado de desarrollo de los países.
En la teoría de Becker, los sistemas educativos son empresas multiproductos por lo que en su análisis hay que buscar el balance entre los beneficios monetarios privados y la ganancia de productividad social. El valor económico de la educación, vista desde la óptica empresarial, junto con el poder de transmisión de ideologías mediante los procesos de enseñanza y aprendizaje constituyen los pilares para que sea utilizada como conductora de ideologías que moldean la voluntad de las sociedades en favor de los intereses del modelo, independientemente de los objetivos culturales y sentimientos morales que el discurso académico plantee como razón fundamental.

El discurso academicista elude el debate sobre la funcionalidad instrumental de la educación superior al aclimatar postulados, ideología y patrones de consumo a la entelequia de pertenecer a la sociedad posmoderna. En eso estriba la efectividad del sistema; por un lado en crear la ilusión en el sujeto de ser libre de mirar la vida desde su relativismo intrínseco y, por otro, lograr que las sumas de individualidades generen la sinergia necesaria para el dominio de todo el tejido social. En consecuencia, los sujetos componentes de la sociedad de consumo masivo quedan atrapados en sus propios deseos, o sea, una prisión social cuyo carcelero es la política corporativa (Botero, 2000).

La educación caballo de Troya, mito o realidad, utilizada como herramienta prometedora de un futuro 
mejor, en la práctica ha sido un instrumento de política económica con la condición sine qua non de ser bendita y profana al mismo tiempo. La memoria histórica de los planes y programas que han recurrido a ella para darles bases a sus promesas, objetivos y metas, ratifican su condición multiuso en los avatares de la sociedad. Educar para lo bueno o lo malo, para el crecimiento y el desarrollo económico, para la tolerancia y la sostenibilidad, en fin, es medicamento esencial para todos los males sociales (Restrepo, 2006).

La Unesco, organismo rector de la política educativa en el mundo, refiriéndose a la educación superior, la encuentra como tratamiento para las aflicciones relacionadas con: erradicar pobreza, intolerancia, violencia, analfabetismo, hambre, deterioro del medioambiente y medicina preventiva de enfermedades. Como se observa, realidad o mito, la educación utilizada como caballo de Troya para introducir ideologías y esperanzas.

La educación, concebida cual principio y fin de derrotas o fracasos o promesa colectiva inconclusa de las categorías de justicia e igualdad en el imaginario social; la educación, límite que separa el oscurantismo y lo profano, lo culto y lo divino. Lo cierto es que su aplicación se ha recomendado con plena certeza para: sacar a los pueblos de la pobreza, dinamizar el crecimiento económico, propiciar el desarrollo social, generar competitividad, afianzar la identidad nacional y la cohesión social. En síntesis, según Jacques Delors (1996) aunque no sea un remedio milagroso, la educación encierra un tesoro (Herrera, 2013).

\section{Conclusiones}

Incursionar en el campo de la economía de la educación es analizar el quehacer académico sometido al itinerario sistémico de producción, distribución y consumo de la inteligencia, donde la educación en este sentido, desempeña el papel de moderna mercancía en la sociedad del conocimiento. Es hacer un análisis holístico de la educación con predominante valor de cambio y develar, sin ambages, el contraste entre la realidad del discurso académico y el carácter utilitarista que el paradigma de mercado imprime a la educación superior.

La retórica sobre los conceptos de autonomía, libertad y autodeterminación crea la sensación de autogobierno, generando un proceso de gubertamentalidad que se retroalimenta de la sensación subjetiva de pertenecer a la sociedad de consumo por 
voluntad propia. De esta manera, la razón económica prima sobre la razón social del Estado. La autonomía universitaria proclamada en la Constitución política y en la ley se cercena frente a los patrones de financiamiento, con lo que se permea la libertad académica en un esquema educativo estadocéntrico en lo administrativo y mercadocéntrico en lo financiero.

Mantener los méritos como estándar de selección, mimetiza la realidad de un Estado con notoria incapacidad para responder a la demanda creciente de educación superior al tiempo que legitima la desigualdad merecida que infaliblemente es una forma sofisticada de discriminación.

En síntesis, la educación superior ha sido utilizada como vehículo para introducir la lógica economicista del modelo de predominio de mercado, a nombre de la pertinencia, se desfiguran las funciones sustantivas de docencia, investigación y proyección social en el proceso de generación de conocimiento, atributo este que es el legítimo parámetro para rendir cuentas a la sociedad.

La historia registra la recurrencia de organismos como el Banco Mundial, el Fondo Monetario Internacional y la Unesco, acudiendo a la educación en su faceta de herramienta social para cerrar las brechas entre lo pobre y lo rico, entre el norte y el sur; en efecto, las cifras o estadísticas sobre cobertura aumentan, pero los niveles de pobreza continúan creciendo. Este fenómeno invita a repensar la educación como política, el análisis historiográfico y las futuras investigaciones aplicadas deben profundizar y dirimir entre si la educación es un medio o en la práctica es un objetivo; si realmente la labor de las universidades se desarrolla en un entorno de autonomía y libertad de cátedra o es solo un sofisma que crea la sensación de autogobierno como estrategia de subordinación solapada.

\section{Referencias bibliográficas}

Agüero, J.O. (2010). Michel Foucault y la gubernamentalidad financiera. Reflexiones sobre la crisis financiera internacional. Revista Visión de Futuro, 14.

Ahumada, C. (1998). El modelo neoliberal. Bogotá: El Áncora Editores.

Banco Mundial (BM) (2000). La educación superior en los países en desarrollo: peligros y promesas. Washington: World Bank.

Becker, G.S. (1983). El capital humano. Un análisis teórico y empí rico referido fundamentalmente a la educación. Madrid: Alianza Editorial.

BOTERO, Uribe Dario. (2000). El derecho a la utopía. Bogotá: Eco

Clark, B. (1997). Universidades modernas: espacios de investigación y docencia. México: Grupo Editorial Miguel Ángel Porrúa.

Cubillos, C. (1998). Saldo en rojo. La educación superior en crisis. Bogotá: Planeta Colombiana Editorial S.A. 
Delors, J. (1996). La educación encierra un tesoro. Informe a la Unesco de la Comisión Internacional sobre la Educación para el siglo XXI.

Foucault, M. (2008). Nacimiento de la biopolítica. Buenos Aires: Fondo de Cultura Económica.

Garay, L. (1999). Globalización y crisis. ¿Hegemonía o corresponsabilidad? Bogotá: Tercer Mundo Editores.

Habermas, J. (1973). Problemas de legitimación en el capitalismo tardío. Buenos Aires: Amorrourtu Editores.

Herrera, J. (2013). Economía de la educación y financiamiento de la educación superior. El ocaso del paradigma social. Universidad de Cartagena 1980-2002. Berlín: Publicia.

Ley 30 de 1992. Ley de educación Superior. Diario oficial, 40700. 29 de diciembre.

Morín, E. (2000). Los sietes saberes necesarios a la educación del futuro. Caracas: Iselac, Unesco.

Paniagua, J. (2003). Latinoamérica y la Institución Libre de Enseñanza de España. Revista Historia de la Educación Latinoamericana, 5, 11-30.

Restrepo, J. (2006). Educar en el atraso social. Bogotá: Planeta.

Rifkin, J. (2000). La era del acceso, la revolución de la nueva economía. Madrid: Paidós.

Sen, A. (2000). Desarrollo y libertad. Bogotá: Planeta.

Shultz, T. (1961). El concepto de capital humano: respuesta. En: M. Blaug. Economía de la educación. Madrid: Tecnos S.A.

Uribe, D. (2000). El derecho a la utopía. Bogotá: Ecoe Ediciones.

Villarroel, C. (2006). Estado y mercado en la educación superior latinoamericana: de la Reforma de Córdoba al Consenso de Washington. Caracas: Impresores Plasarte C.A.

Veiga-Neto, A. (2010). Gubernamentalidad neoliberal: implicaciones para la educación. Revista Educación y Pedagogía, 22, 213-235. 